\title{
Study of Serum Calprotectin Level in Patients with Acne Vulgaris and its Correlation to Disease Severity
}

\author{
I.Y.Abd Allah ${ }^{1}$, M.A.Gab Allah ${ }^{2}$, D.S.Abdel Salam ${ }^{2}$ and A.M.El Sahafy ${ }^{1}$ \\ ${ }^{1}$ Dermatology, Venereology and Andrology,Dept., Faculty of Medicine, Benha Univ., Benha, Egypt \\ ${ }^{2}$ Medical Biochemistry,Dept., Faculty of Medicine, Mansoura Univ., cairo, Egypt \\ Email: ElSahafyAhmed @yahoo.com
}

\begin{abstract}
Acne vulgaris is a common skin condition that affects people all over the world. Acne vulgaris is characterised by the presence of open and closed comedones, papules, pustules, and nodules on areas of the body with hormonally sensitive sebaceous glands. Acne is thought to be caused by four key factors: increased sebum production, follicular hyperkeratinization, skin bacterial colonisation, and inflammation. Acne with severe symptoms is painful, disfiguring, scarring, and lowering self-esteem. The aim of this study was to determine the serum level of calprotectin in acne vulgaris patients and its relationship to disease severity. Subjects and Methods: In this sample, 60 people with acne vulgaris were paired with 30 people who appeared to be of similar sex and age as a control group. The patients were chosen from those who attended the Benha University Hospital's Dermatology and Andrology Department's Outpatient Clinic between April 2019 and October 2020. Our findings indicated that the AV group had a statistically significant higher level of serum calprotectin with mean SD (6.291.91ng/dl) than the control group with mean SD (2.040.65ng/dl) (p=0.004). The amount of serum calprotectin had a statistically significant positive relationship with the GAGS score $(\mathrm{r}=0.854$; 0.001$)$. The period of the disease, however, had no meaningful correlation $(\mathrm{r}=0.163 ; \mathrm{p}=0.212)$. Conclusion: Serum calprotectin levels are higher in acne vulgaris patients relative to safe controls, and there is a statistically significant relationship between calprotectin levels and disease severity, suggesting that calprotectin may be used as a chemical biomarker to determine disease severity.
\end{abstract}

Keywords: Calprotectin, growth hormone, pathogen-associated molecular patterns.

\section{Introduction}

Acne vulgaris is a skin condition that affects people all over the world. Open and closed comedones, papules, pustules, and nodules are all characteristics of acne. Acne vulgaris typically affects the face, abdomen, stomach, upper back, and upper arms, as well as other areas of the body with hormonally activated sebaceous glands. Severe acne causes inflammation, disfigurement, and scarring, as well as lowering self-esteem and negatively impacting mental wellbeing in certain patients [19].

Based on the amount and form of skin lesions, acne is graded as mild, moderate, or extreme. There are a variety of therapeutic agents and formulations available, each focusing on a different aspect of acne pathogenesis [16].

Acne affects 85 to $90 \%$ of adolescents, as well as children aged 7 to 12 years old and adults, particularly those with darker skin. Adults with acne are more likely to be in their twenties than in their thirties, with 64 percent in their twenties and 43 percent in their thirties. Women are more likely than men to be affected. Acne is thought to influence the majority of people at some stage in their lives, and it is especially prevalent in the Middle East. Although acne is commonly thought to be a selflimiting adolescent disorder, a growing number of adults are suffering from the condition [9].

Acne vulgaris pathogenesis is still a work in progress for us. Many causes, such as hereditary, biochemical, inflammatory, and environmental effects, are believed to have an effect on acne pathophysiology [3].

Increased sebum formation, follicular hyperkeratinization, skin bacterial invasion, and inflammation are thought to all play a role in the development of acne [4].

Our interpretation of Cutibacterium acnes' function in the pathophysiology of acne has recently shifted: acne is caused by a lack of equilibrium between various $C$. acnes phylotypes, as well as a dysbiosis of the skin microbiome. The lack of phylotype variation in C. acnes activates the innate immune response, resulting in cutaneous inflammation [5].

Calprotectin is a calcium binding protein with antimicrobial properties that belongs to the S100 family of leukocyte proteins. It is secreted mainly by neutrophilic granulocytes and monocytes. Calprotectin can chelate the transition metals manganese and zinc when they are present in the presence of calcium. The antimicrobial effects are a product of the metal sequestration. Calprotectin is the only manganese sequestration protein complex that has been identified as antimicrobial [14].

Rheumatoid arthritis [24], Crohn's disease [18], and psoriasis [21] have also been linked to calprotectin. Serum calprotectin levels were shown to be higher in patients with acne vulgaris, suggesting that calprotectin may be a useful parameter in determining the magnitude of acne vulgaris [13].

\section{Aim of the Work}

The aim of this analysis is to determine the serum level of calprotectin in acne vulgaris patients and its relationship to disease severity.

\section{Subjects And Methods}

This research was conducted as a retrospective case analysis sample. 
The Research Ethics Committee of Benha University's Faculty of Medicine gave their approval to this report. Any participant in this research gave his or her informed consent.

The participants in this sample were 60 people with acne vulgaris and 30 people who were sex and age balanced and acted as a control group. The patients were chosen from those who attended the Benha University Hospital's Dermatology and Andrology Department's Outpatient Clinic between April 2019 and October 2020.

\subsection{Subjects}

Inclusion criteria: All patients enrolled in the study were over 15 years old, and had different clinical variants \& degree of severity of acne vulgaris.

\subsection{Exclusion criteria}

All patients with any of the following conditions were excluded from this study: Patients with mental illness, or concurrent physical illness and disabilities that can affect their mental status. Patients with any disease, which compromised quality of life or self-esteem. Patients who failed to answer two or more questions from validated questionnaires. Patients who did not

Table (1) Acne-prone areas and the factor for each area (5) allow researchers to perform the dermatological physical examination.

Each participant was subjected to the following: Full history taking: including personal history, history of other diseases or drug intake. Complete general examination obtaining all relevant clinical data e.g. height, body weight, blood pressure and body mass index (BMI). Body mass index (BMI) is a statistical index using a person's weight and height to provide an estimate of body fat in males and females of any age. It is calculated by taking a person's weight, in kilograms, divided by their height, in meters squared, or BMI = weight (in $\mathrm{kg}$ )/ height ${ }^{\wedge} 2$ (in $\left.\mathrm{m}^{\wedge} 2\right)^{[6]}$. Normal weight BMI greater than or equal to 18.5 to $24.9 \mathrm{~kg} / \mathrm{m}^{\wedge} 2$. Overweight - BMI greater than or equal to 25 to 29.9 $\mathrm{kg} / \mathrm{m}^{\wedge} 2$. Obesity - BMI greater than or equal to 30 $\mathrm{kg} / \mathrm{m}^{\wedge} 2$. Complete cutaneous examination to evaluate clinical types and severity of acne vulgaris.

The clinical severity of acne was assessed by the Global Acne Grading System score (GAGS) (5), it is a numerical grading system based on the acne lesion types and locations Table (1).

This scoring system divides the acne-prone areas into six regions (five on the face and one on the chest/upper back) and gives each an area factor.

\begin{tabular}{ll}
\hline Location & Factor \\
\hline Forehead & 2 \\
Right cheek & 2 \\
Left cheek & 2 \\
Nose & 1 \\
Chin & 1 \\
Chest and upper back & 3 \\
\hline
\end{tabular}

\subsection{Calculation}

The overall severity score was calculated by combining six regional subscores, each of which was calculated by multiplying the component for each area by the most highly weighted lesion in that region: 1 comedone $=1$ comedone 1 papule $=2$ papules $=2$ papules $=2$ papules $=2$ pa 3 pustules $=3$ pustules $=3$ pustules $=3$ pustules $=34$ with each nodule

Surface ground, distribution, and density of pilosebaceous units were used to calculate regional factors. The severity was classified as mild if the score was $1-18$, reasonable if it was 19-30, extreme if it was 31-38, and extremely severe if it was more than 38 [5]. The enzyme-linked immunosorbent assay (ELISA) was used to determine the amount of serum calprotectin $(\mathrm{ng} / \mathrm{mL})$ [15]. Each participant in this study had $3 \mathrm{~mL}$ of blood drawn from them. Sera were isolated, split into two aliquots, and held at -20 degrees Celsius.

Calprotectin was applied to monoclonal antibody enzyme wells that had been pre-coated with biotinlabeled human calprotectin monoclonal antibodies and mixed with Streptavidin-HRP to create an immune complex, accompanied by another incubation and washing to eliminate the uncombined enzyme. After that, applying chromogen solutions $\mathrm{A}$ and $\mathrm{B}$ causes the liquid to become blue in colour. As a consequence of the acid being applied, the colour gradually became yellow. The abundance of the human material calprotectin in tests was positively associated with the colour chroma. The findings were obtained after plotting the normal curve (Human Calprotectin (CALPRO) ELISA Kit).

\subsection{Assay Procedure}

In standard wells: $50 \mu 1$ of standard and $50 \mu 1$ of streptavidin-HRP were added (as the standard already contains combined biotin antibody, adding the antibody is not mandatory). In test wells: after adding $40 \boldsymbol{\mu} 1$ of the sample, we added both $10 \boldsymbol{\mu} 1$ of CALPRO antibody and $50 \mu 1$ of streptavidin-HRP. Then sealing membrane was sealed and gently shaked then incubated for one hour at $37^{\circ} \mathrm{C}$. $30 \mathrm{X}$ wash buffer was diluted 30 times using distilled water. After carefully removing the membrane liquid was drained and the remaining water was removed. Then $50 \mu 1$ of chromogen solution B were added to the wells. Gently mixed, incubated for 10 minutes at $37^{\circ} \mathrm{Cin}$ a dark place. A $50 \mu \mathrm{l}$ of stop solution were added in every well for stopping the reaction (instantly changing the blue color to yellow one). The optical density was measured using wave length of 450 $\mathrm{nm}$ and measuring was done withen 15 minutes. As 
regard the standards, concentration and the corresponding optical density values, we calculated the standard curve using linear regression equation, afterward we applied sample optical density values on the regression equation to calculate the equivalent concentration of samples. Some computer software are available to make the calculations.

\subsection{Calculation}

Using the standard density as the horizontal line, the optical density value as the vertical, the standard curve was drawn on graph paper. Doing that, we can obtain the corresponding concentration of the sample from the standard curve.

\subsection{Statistical Analysis}

The collected data was revised, coded, tabulated and introduced to a PC using Statistical package for Social Science (IBM Corp. Released 2017. IBM SPSS Statistics for Windows, Version 25.0. Armonk, NY: IBM Corp.). Data were presented and suitable analysis was done according to the type of data obtained for each parameter.

Normality of data: Shapiro test was done to test the normality of data distribution.

Descriptive statistics: Mean, Standard deviation $( \pm$ SD) for parametric numerical data, median and range for non parametric numerical data. Frequency and percentage of non-numerical data.

Analytical statistics: Student $\mathrm{T}$ Test was used to assess the statistical significance of the difference between two study group means. For the comparison of the three groups' means, one way analysis of variance (ANOVA) was used. Chi-Square test was used to examine the relationship between two qualitative variables. Fisher's exact test: was used to examine the relationship between two qualitative variables when the expected count is less than 5 in more than $20 \%$ of cells. Correlation analysis: To assess the strength of association between two quantitative variables. The correlation coefficient defines the strength and direction of the linear relationship between two variables. The ROC Curve (receiver operating characteristic) provides a useful way to evaluate the sensitivity and specificity for quantitative diagnostic measures that categorize cases into one of two groups. The optimum cut off point was defined as that which maximized the AUC value. The area under the ROC curve (AUC) results were considered excellent for AUC values between 0.9-1, good for AUC values between 0.8-0.9, fair for AUC values between $0.7-0.8$, poor for AUC values between 0.6-0.7 and failed for AUC values between 0.5-0.6 ${ }^{[12]}$. Regression analysis: Logistic and linear regression analyses were used for prediction of risk factors, using generalized linear models. Odds ratio and 95\% confidence interval were calculated. Beta regression coefficient describes the relationship between a predictor variable and the response. The sign of beta coefficient indicates the direction of the relationship. The coefficient value represents the mean change in the response given a one unit change in the predictor. If the p-value for a variable is $<0.05$, data provide enough evidence of correlation, and changes in the independent variable are associated with changes in the response. A p value is considered significant if $<0.05$ at confidence interval $95 \%$.

\section{Results}

Table (1) Comparison of demographic data between cases and control groups.

\begin{tabular}{|c|c|c|c|c|c|c|}
\hline & & Control & $n=30$ & $\mathbf{A V}$ & $n=60$ & p \\
\hline Age (years) & Mean \pm SD & 22.7 & \pm 3.6 & 21.4 & \pm 4.2 & 0.154 \\
\hline Males & n, $\%$ & 5 & $16.7 \%$ & 9 & $15.0 \%$ & 0837 \\
\hline Females & $\mathrm{n}, \%$ & 25 & $83.3 \%$ & 51 & $85.0 \%$ & 0.851 \\
\hline
\end{tabular}

$\mathrm{SD}$, standard deviation; student $\mathrm{t}$ test was used for numerical parameters; Chi square test was used for categorical parameters; $\mathrm{p}<0.05$ is considered significant.

Table (2) Comparison of anthropometric data between cases and control groups.

\begin{tabular}{|c|c|c|c|c|c|c|}
\hline & & Control & $n=31$ & $\mathbf{A V}$ & $n=60$ & p \\
\hline BMI & mean \pm SD & 27.3 & \pm 4.3 & 26.1 & \pm 5.9 & 0.333 \\
\hline Non obese & $\mathrm{N}, \%$ & 19 & $63.3 \%$ & 48 & $80.0 \%$ & \multirow{2}{*}{0.187} \\
\hline Obese & $\mathrm{N}, \%$ & 11 & $36.7 \%$ & 12 & $20.0 \%$ & \\
\hline
\end{tabular}

$\mathrm{SD}$, standard deviation; Student $\mathrm{t}$ test was used for numerical parameters; Chi square test was used for categorical parameters; $\mathrm{p}<0.05$ is considered significant.

Table (3) Clinical features in all studied AV cases.

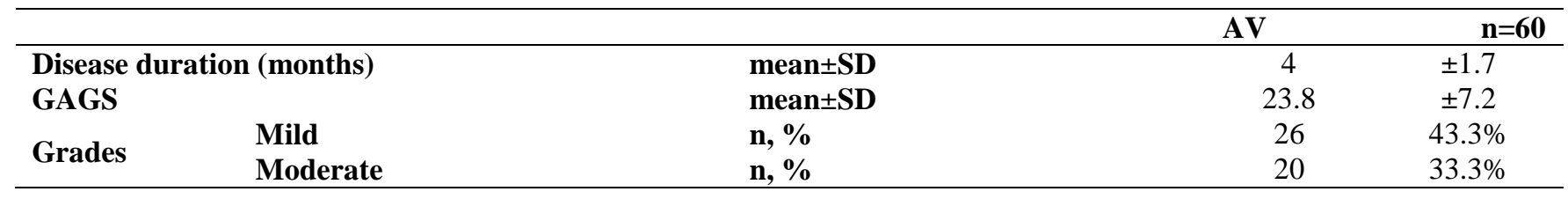




\begin{tabular}{llcc} 
Severe & $\mathbf{n}, \%$ & 9 & $15.0 \%$ \\
Very severe & $\mathbf{n}, \%$ & 5 & $8.3 \%$ \\
\hline
\end{tabular}

Table (4) Comparison of serum calprotectin level among studied groups.

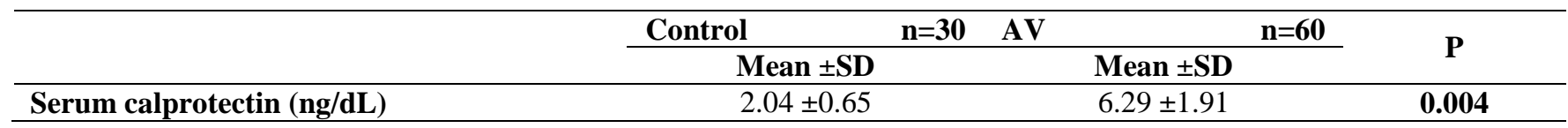

$\mathrm{SD}$, standard deviation; $\mathrm{p}<0.05$ is considered significant.

Table (5) Validity of serum calprotectin level for discrimination between AV cases and control groups.

\begin{tabular}{ll}
\hline & Serum Calprotectin \\
\hline AUC & 0.713 \\
Cut off & 2.6 \\
Sensitivity (\%) & 50 \\
Specificity (\%) & 83.3 \\
PPV (\%) & 50 \\
NPV $(\%)$ & 83.3 \\
Accuracy $(\%)$ & 61.1 \\
\hline
\end{tabular}

AUC, area under ROC, ROC, receiver operating curve; PPV, positive predictive value; NPV, negative predictive value.

Table (6) Validity of Serum Calprotectin level for prediction of AV severity.

\begin{tabular}{ll}
\hline & Serum Calprotectin \\
\hline AUC & 0.954 \\
Cut off & 3.9 \\
Sensitivity (\%) & 100 \\
Specificity (\%) & 80.4 \\
PPV (\%) & 100 \\
NPV (\%) & 80.4 \\
Accuracy (\%) & 85 \\
\hline
\end{tabular}

AUC, area under ROC, ROC, receiver operating curve; PPV, positive predictive value; NPV, negative predictive value.

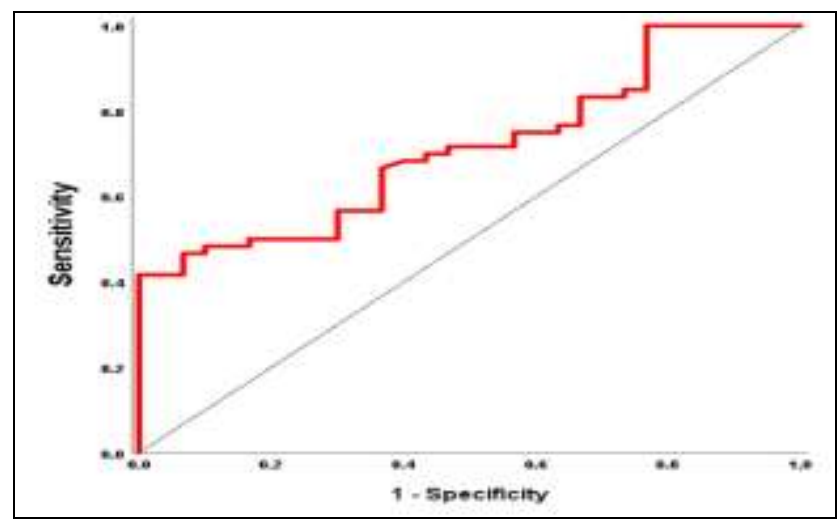

Fig. (1) ROC curve of serum calprotectin level for discrimination between AV cases and control groups.

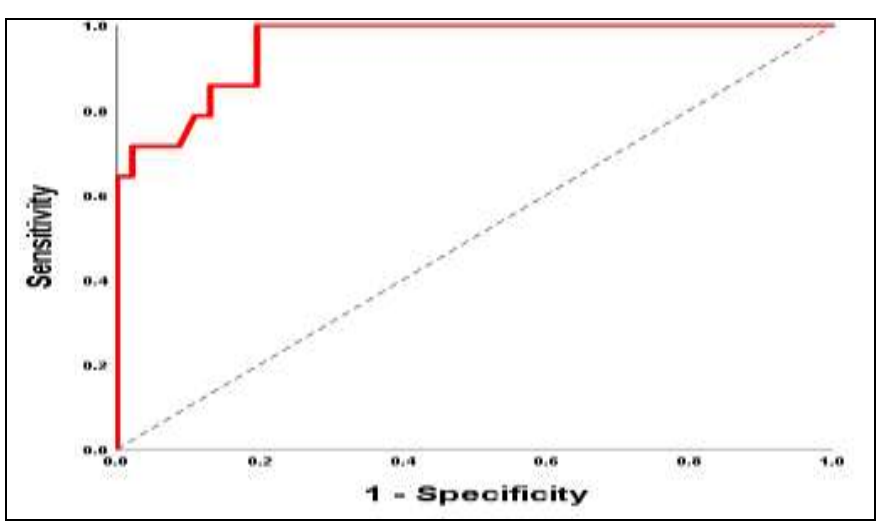

Fig. (2) ROC curve of serum calprotectin level for prediction of AV severity.

Table (7) Association of serum calprotectin level according to gender and Nutritional status in control group.

\begin{tabular}{|c|c|c|c|c|c|c|}
\hline & & \multicolumn{4}{|c|}{ Serum Calprotectin } & \multirow{2}{*}{$\mathbf{p}$} \\
\hline & & $\mathbf{n}$ & mean & \pm & SD & \\
\hline \multirow{2}{*}{ Gender } & Male & 5 & 2.24 & \pm & 0.70 & \multirow{2}{*}{0.458} \\
\hline & Female & 25 & 2 & \pm & 0.63 & \\
\hline \multirow{2}{*}{$\begin{array}{l}\text { Nutritional } \\
\text { status }\end{array}$} & Non obese & 19 & 1.94 & \pm & 0.61 & \multirow{2}{*}{0.295} \\
\hline & Obese & 11 & 2.21 & \pm & 0.60 & \\
\hline
\end{tabular}


$\mathrm{SD}$, standard deviation; student $\mathrm{t}$ test was used for numerical parameters; $\mathrm{p}<0.05$ is considered significant.

Table (8) Association of serum calprotectin level according to gender, nutritional status and grades of AV group.

\begin{tabular}{|c|c|c|c|c|c|c|}
\hline & & \multicolumn{4}{|c|}{ Serum Calprotectin } & \multirow[b]{2}{*}{$\mathbf{p}$} \\
\hline & & n & mean & \pm & SD & \\
\hline \multirow{2}{*}{ Gender } & Male & 9 & 5.39 & \pm & 1.08 & \multirow{2}{*}{0.713} \\
\hline & Female & 51 & 6.45 & \pm & 1.42 & \\
\hline \multirow{2}{*}{ Type } & Non obese & 48 & 6.01 & \pm & 1.56 & \multirow{2}{*}{0.591} \\
\hline & Obese & 12 & 7.40 & \pm & 2.45 & \\
\hline \multirow{4}{*}{ Grades } & Mild & 26 & 1.83 & \pm & 0.34 & \multirow{4}{*}{$<0.001$} \\
\hline & Moderate & 20 & 4.81 & \pm & 1.20 & \\
\hline & Severe & 9 & 11.88 & \pm & 3.08 & \\
\hline & Very severe & 5 & 25.31 & \pm & 7.24 & \\
\hline
\end{tabular}

$\mathrm{SD}$, standard deviation; student t test was used for numerical parameters comparing 2 groups; ANOVA was used for numerical parameters comparing more than 2 groups $\mathrm{p}<0.05$ is considered highly significant.

Table (9) Correlations of serum calprotectin level with age and BMI in control group.

\begin{tabular}{lcc}
\hline & \multicolumn{3}{c}{ Serum Calprotectin } \\
\cline { 2 - 4 } & $\mathbf{R}$ & $\mathbf{P}$ \\
\hline age & -0.028 & 0.884 \\
BMI & 0.310 & 0.096 \\
\hline
\end{tabular}

$\mathrm{r}$, correlation coefficient; positive $\mathrm{r}$ values indicate positive correlation; negative $\mathrm{r}$ values indicate negative correlation; $\mathrm{p}<0.05$ is considered significant.

Table (10) Correlations of serum calprotectin level with age, BMI duration and GAGS score in AV group.

\begin{tabular}{lcc}
\hline & \multicolumn{2}{c}{ Serum Calprotectin } \\
\cline { 2 - 3 } & $\mathbf{R}$ & $\mathbf{p}$ \\
\hline Age & 0.082 & 0.535 \\
BMI & 0.038 & 0.772 \\
Duration & 0.163 & 0.212 \\
GAGS & 0.854 & $<0.001$ \\
\hline
\end{tabular}

r, correlation coefficient; positive $r$ values indicate positive correlation; negative $r$ values indicate negative correlation; $\mathrm{p}<0.05$ is considered significant.

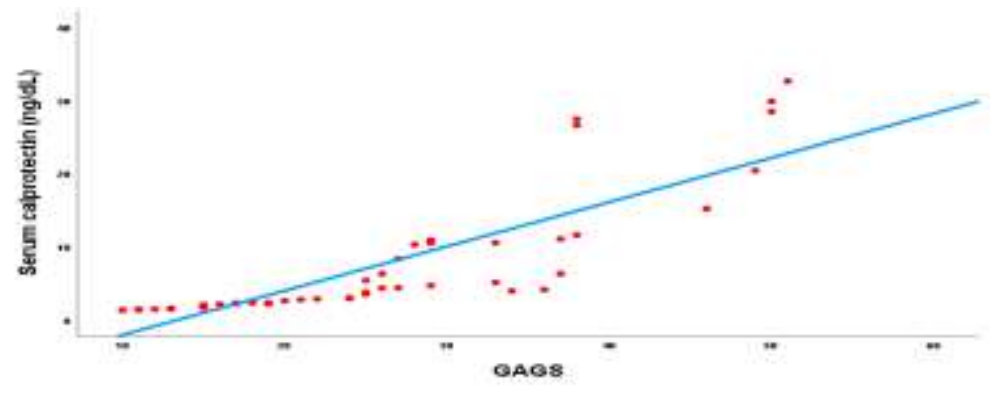

Fig. (3) Correlations of serum calprotectin level with GAGS score in AV group.

Table (11) Regression analysis for prediction of AV susceptibility.

\begin{tabular}{lllll}
\hline & $\mathbf{p}$ & OR & $\mathbf{9 5 \%}$ CI & \\
\hline Age & 0.148 & 0.951 & 0.889 & 1.018 \\
Gender & 0.838 & 1.080 & 0.519 & 2.248 \\
BMI & 0.209 & 0.966 & 0.914 & 1.020 \\
Calprotectin & 0.017 & 1.511 & 1.077 & 2.119 \\
\hline
\end{tabular}


OR, odds ratio; $\mathrm{CI}$, confidence interval. Logistic regression test was used; $\mathrm{p}<0.05$ is considered significant; OR $<1$ indicates protective effect; OR>1 indicates risky effect.

Table (12) Regression analysis for prediction of factors affecting severity of AV (higher GAGS score).

\begin{tabular}{lcc}
\hline & $\boldsymbol{\beta}$ & $\mathbf{p}$ \\
\hline Age & 0.25 & 0.460 \\
Gender & 1.47 & 0.713 \\
BMI & 0.14 & 0.280 \\
Duration & 0.52 & 0.321 \\
Calprotectin & 1.21 & $<0.001$ \\
\hline
\end{tabular}

$\mathrm{B}$, linear regression coefficient; linear regression test was used; $\mathrm{p}<0.05$ is considered highly significant; negative $\mathrm{B}$ value indicates decreasing effect on GAGS; positive B value indicates increasing effect on GAGS.

\section{Discussion}

Acne vulgaris is a widespread chronic inflammatory skin disease affecting the pilosebaceous unit that has a multifactorial pathogenesis, a wide range of anatomy, and a significant psychological effect. The appearance of open and closed comedones, papules, pustules, and nodules on the forehead, throat, stomach, upper back, and upper arms characterises it [2].

Acne vulgaris affects an estimated 9.4 percent of the world's population, with teens having the greatest incidence. In 12 to 14 percent of adults, acne persists into adulthood, triggering extreme psychosocial dysfunction [3].

Calprotectin is a leukocyte protein that is secreted mainly by neutrophilic granulocytes and monocytes and belongs to the S100 band. It's the only antimicrobial manganese sequestration protein complex that's been discovered [14]. Calprotectin, a new blood-based biomarker, has been the subject of a lot of recent research. Many inflammatory diseases and conditions, including skin diseases such as psoriasis [12] and acne vulgaris [13], have been linked to CLP.

The aim of this research was to determine the amount of serum calprotectin in acne vulgaris patients, as well as the relationship between calprotectin serum level and disease intensity. Our research involved 60 acne vulgaris patients (patients) and 30 seemingly safe people who were matched for sex, age, and BMI as a control group.

The bulk of the AV patients we examined were females, according to our findings (85 percent ). This finding was similar to those seen in [12] and [8]. The rest of their patients were women, according to them (66.07 percent, 84.3 percent and 75 percent respectively). This discovery may be attributed in part to females' greater concern about their appearance and attractiveness, prompting them to pursue medical advice more often than males.

Mild acne was found to be the most prevalent clinical form (43.3 percent) among the patients surveyed, accompanied by moderate and heavy acne. In conjunction with [11] and [1], this was done. Mild acne was the most prevalent form among the patients surveyed, according to the researchers (60.2 percent, 53 percent and 64 percent respectively).

In our sample, $20 \%$ of the patients were overweight. In conjunction with [17], this was done.
They discovered no connection between having a higher BMI and having acne vulgaris. However, [10] found that overweight and obesity are linked to acne in girls aged 18 and 19, but not in youth, in their research.

The mean amount of serum calprotectin in our sample was $6.29 \mathrm{ng} / \mathrm{dl}$ in patients and $2.04 \mathrm{ng} / \mathrm{dl}$ in controls, with a statistically meaningful improvement in AV patients relative to controls (p0.05). Our findings were consistent with those of [7], who discovered a substantial rise in serum calprotectin levels in their AV patients as opposed to the control group, despite the fact that the discrepancy was extremely statistically significant (P value 0.001). Furthermore, our findings were consistent with those of [13]. They discovered that the amount of serum calprotectin in acne vulgaris patients was statistically substantially higher than in stable samples.

The amount of serum calprotectin was found to have a strongly important positive association with GAGS score $(r=0.854$; 0.001$)$ in our research. This matched the findings of [7], who discovered a strongly important positive association between serum calprotectin levels and acne incidence in their case community. [13], on the other hand, did not find this important connection.

Our findings, on the other hand, revealed no important connection between calprotectin serum levels and the length of AV in our patients. This contrasted with the findings of [7], who discovered a strong positive association between serum calprotectin levels and acne length.

Calprotectin can act as an accurate predictor of acne intensity, according to our results, and may aid in the care and follow-up of patients with acne vulgaris.

\section{Conclusion}

Calprotectin levels in the blood are higher in acne vulgaris patients than in stable control groups, and they have a statistically significant relationship with disease intensity, suggesting that calprotectin may be used as a chemical biomarker to determine disease severity.

\section{References}

[1] M.Alanazi, H.Hammad , A.Mohamed , Prevalence and psychological impact of Acne vulgaris among female secondary school students in Arar city, Saudi 
Arabia. Electronic Physician; vol.10(8) ,pp. 7224$7229,2018$.

[2] L.Al-Kathiri , T.Al-Najjar , Severe Nodulocystic Acne not responding to Isotretinoin Therapy Successfully Treated with Oral Dapsone. Oman Med J;vol. 33(5),pp. 433-436,2018.

[3] H. Baldwin , J. Tan , Effects of Diet on Acne and Its Response to Treatment. Am J Clin Dermatol; vol.10(20) ,pp. 257-542,2020.

[4] E. Barnard, B. Shi, D. Kang, N. Craft , H. Li, The balance of metagenomic elements shapes the skin microbiome in acne and health. Sci Rep;vol.6,pp.3949,2016.

[5] Doshi, A. Zaheer , M.J. Stiller, "A comparison of current acne grading systems and proposal of a novel system." International journal of dermatology;vol. 36(6),pp.416-418,1997.

[6] B .Dréno, E .Araviiskaia, D .Kerob, Nonprescription acne vulgaris treatments: Their role in our treatment armamentarium-An international panel discussion.J Cosmet Dermatol;vol.19,pp.2201-2211,2020.

[7] M.A. El-Hamd, E.E.A. Nada, M.A .Moustafa , R.A. Mahboob-Allah, Prevalence of acne vulgaris and its impact of the quality of life among secondary schoolaged adolescents in Sohag Province, Upper Egypt. J Cosmet Dermatol;vol. 16(3),pp.370-373,2017.

[8] I .Fouda, Z .Obaid, S. Hegazy, H. Abd Al-Samie, A. Nofal , Calprotectin in acne vulgaris: A possible contributory role. J Cosmet Dermatol;vol.5(3), pp.15,2020.

[9] K. França , J. Keri , Psychosocial impact of acne and post inflammatory hyperpigmentation. An Bras Dermatol; vol.92(4),pp. 505-509,2017.

[10] M.H. Gold, D.J. Goldberg, M.S. Nestor , Current treatments of acne: Medications, lights, lasers, and a novel 650-1s 1064-nm Nd: YAG laser. J Cosmet Dermatol;vol. 16,pp.303-318,2017.

[11] J .Halvorsen, R. Vleugels, E. Bjertness, A Population-Based Study of Acne and Body Mass Index in Adolescents. Arch Dermatol;vol.148(1),pp.131-132,2012.

[12] N .Hazarika , M .Archana , The psychosocial impact of acne vulgaris. Indian Journal of Dermatology;vol. 61(5),pp.515-520,2016.

[13] K. Ismail , K .Mohammed-Ali , Quality of life in patients with acne in Erbil city. Health and Quality of Life Outcomes;vol.54,pp. 10-60,2012.

[14] S. Korkmaz , S.K. Fiçıcıoğlu ,Calprotectin can play an inflammatory role in acne vulgaris. Adv Dermatol and Alergol;vol. 35 (4),pp.397-399,2018.

[15] F.S. Lehmann, E. Burri, C. Beglinger, The role and utility of faecal markers in inflammatory bowel disease. Ther Adv Gastrointest;vol. 8 (1),pp.2336,2014 .

[16] R.M. Lequin , Enzyme immunoassay (EIA):/enzyme-linked immunosorbent assay (ELISA): Clinical chemistry;vol. 51(12),pp. 2415-2418,2005.

[17] K.Linda Ogé, Alan Broussard, D.Marilyn Marshall, Acne Vulgaris: Diagnosis and Treatment. Am Fam Physician;vol. 100(8),pp.475-484,2019.
[18] C. Mazzella, G. Fabbrocini, M. Luciano, S .Cacciapuoti, Nevus Comedonicus With Acne Vulgaris and Obesity. Dermatol Pract Concept;vol. 10(1),pp. 202-6,2019.

[19] M.A. Meuwis, G. Vernier-Massouille, J.C. Grimaud, Serum calprotectin as a biomarker for Crohn's disease. Journal of Crohn's and Colitis;vol. 7(12),pp. 678-83,2013.

[20] A.M. O’Neill , R.L. Gallo , Host-microbiome interactions and recent progress into understanding the biology of acne vulgaris. Microbiome; vol.6(1),pp.177-175,2018.

[21] J. Perić, N. Maksimović, J. Janković, B. Mijović, V. Reljić , S. Janković , Prevalence and quality of life in high school pupils with acne in Serbia. Vojnosanit Pregl; VOL.70(10),PP.935939,2013.

[22] M. Qian , N.J. Song , Serum calprotectin correlates with risk and disease severity in psoriasis patients and the decrease of calprotectin predicts better response to tumor necrosis factor inhibitors. Eur Rev Med Pharmacol Sci; vol.22 (13),pp.42994309,2018.

[23] T. Stewart, C. Bazergy , Hormonal and dietary factors in acne vulgaris versus controls. Dermatoendocrinol; vol.10(1),pp. 144-160,2018.

[24] J.K. Tan , K. Bhate , A global perspective on the epidemiology of acne. Br. J. Dermatol; vol. 172(1),pp.3-12,2015.

[25] Q. Wang, W. Chen, J. Lin ,The Role of Calprotectin in Rheumatoid Arthritis. J Transl Int Med; vol.7(4),pp.126-131,2019. 